\title{
Mashups'07: First International Workshop on Web APIs and Services Mashups
}

\author{
E. Michael Maximilien ${ }^{1}$ and Stefan Tai ${ }^{2}$ \\ ${ }^{1}$ IBM Research \\ ${ }^{2}$ Karlsruhe Institute of Technology (KIT)
}

\section{Introduction}

Services computing and Web 2.0 computing are converging into a programmable Web today. However, the interaction and integration of the two classes of technologies and approaches expose various issues and complexities that have to be addressed, especially as these new programmable Web solutions displace current legacy distributed system applications and middleware. Mashups'07 is an international workshop intended to foster a conversation around the challenges and issues that the services computing community is facing as the Web 2.0 technologies, approaches, and techniques and the Web services more mature approaches merge to help bring about the innovation potentials of the programmable Web. The following pages constitute the post-proceedings of Mashups'07 - the inaugural workshop in an anticipated series of workshops that look specifically at the engineering, scientific, social, and business concerns and challenges encountered from end-user-oriented compositions of Webaccessible services, APIs, and content, that is of Web mashups.

Mashups'07 was held as a one-day workshop on September 13, 2007, co-located with ICSOC in Vienna, Austria. The workshop's call for papers targeted submissions on topics spanning:

- Programming models (languages, frameworks, and platforms) for the composition of Web-accessible services, APIs, content, and data of all kinds and of different architectural styles (e.g., SOAP, REST, RSS, Atom, and Atom Publishing Protocol).

- Quality of mashups, including reliability and security, and management approaches.

- Understanding social and economic factors in the creation, acceptance, and sustainability of services mashups, including software-as-services (SaaS) markets and services marketplaces, digital communities, as well as directory, pricing, and contracting models.

\section{Program}

The Mashups'07 program consisted of one keynote presentation, three invited papers, a panel session, and a social program ${ }^{1}$. Participants of the workshop represented

\footnotetext{
${ }^{1}$ http://maximilien.org/workshops/mashups/2007/ 
several relevant communities: application (mashup) developers, mashup technology developers, providers, and experts, end-users, social networking and economics researchers, as well as the broader services computing community.

The keynote presentation was by Duane Nickull, Senior Technical Evangelist at Adobe Systems. Duane gave an introspective analysis of SOA and Web 2.0, and suggested an "Enterprise Mashup Architecture" that builds on the foundations of SOA. In particular Duane tried to distill and structure current Web mashups techniques into reusable patterns and suggested how to blend SOA techniques into his growing patterns catalog. Duane's slides are available at the workshop's Web site. The keynote presentation was followed by a research paper session that featured three invited papers ranging from a pure engineering exposé of mashups techniques, to a data flow research-oriented paper, and finally to a business analysis paper on emerging Web service and mashups marketplace. The three invited papers were peer-reviewed by an international program committee consisting of experts from both industry and academia. The Mashups'07 panel was moderated by Stefan Tai (Karlsruhe University) and featured expert statements by Gregor Hohpe (Google, Inc.), Duane Nickulls (Adobe, Inc.), E.M. 'max' Maximilien (IBM Almaden Research Center), and Christine Legner (University of St. Gallen). The prepared statements were followed by a lively discussion with the workshop participants. A set of "seed questions" were raised to the panelists, which can be found at the workshop's Web site. After the allotted time for the panel's questions and answers, the workshops' attendees and panelists continued the discussion at a local Viennese restaurant for lunch and further discussions.

\section{Conclusion}

Mashups'07 was a successful workshop dedicated to the exchange of research results, practitioners' experiences, and discussion of trends for a very dynamic and emerging area within Web and Services computing. Recent investments by industry leaders into mashups tools and platforms such as Yahoo! Pipes, Google Mashups Editor, as well IBM's Mashups Hub, QEDwiki, DAMIA, and Sharable Code, is strong evidence that the mashups marketplace is gaining a strong foothold and continues to gain the imagination of services computing customers. While mashups are being used now to solve situational problems in enterprises and empowering business users and endusers, the many questions and discussions raised by Mashups'07 audience, panelists, and authors point to the fact that there are still many engineering and social issues to be resolved. Mashups'07 was a first step toward an open discussion bridging the twin communities of services computing and Web 2.0. Together we hope to continue make some progress toward realizing the common vision and purpose of transforming the Web into a programmable substrate for business and society.

The organizers would like to thank Duane Nickull, the papers' authors, the panelists, the workshop participants, and the international program committee for contributing to the workshop. We are grateful to our sponsors Adobe Systems, IBM Research, and StrikeIron for their financial support. Finally, we would like to thank the ICSOC conference organizers for supporting Mashups'07. 